\title{
The cD Galaxy in Abell Cluster 1775
}

\author{
J. J. E. Hayes and B. Bhattacharya \\ Space Telescope Science Institute \\ 9700 San Martin Drive \\ Baltimore, MD 21218
}

\begin{abstract}
Over the last 20 years, a number of workers have studied the multiple nuclei $\mathrm{cD}$ galaxy in the rich Abell cluster 1775, trying to discover its nature. In all the cases though, very little has been published concerning its morphology. The majority of arguments about the nature of the this object have been based on the relative radial velocities of the 2 components with each other and with the other galaxies in the cluster, or its radio morphology. Very little work has been done on the optical morphology. To rectify that lack of data, we have obtained BVRI CCD images of the $\mathrm{CD}$. We find from our CCD data that the $\mathrm{CD}$ is unlikely to be a bound object and that there is strong evidence for an collision.
\end{abstract}

\section{Introduction}

The $\mathrm{cD}$ system in Abell cluster 1775 has been studied by a number of workers in the past. Chincarini et al. (1971), using Palomar Schmidt plates and image-tube spectra, argued that this object was a gravitationally bound pair, forming a stable system having a mass of a few times $10^{13}$ solar masses. They noted that the pair was in a sparse region of the cluster and that the colours of both components were "normal" for ellipticals. The conclusion was that such an isolated system which appeared to be undisturbed would be a stable, bound system. However, Hintzen (1979) using his original spectroscopy confirmed that the relative velocities of the two galaxies were $\approx 1700 \mathrm{~km} \mathrm{~s}^{-1}$ and noted the discovery by Miley and Harris (1977) of a 300 kpc long straight radio-tail emerging from the SE galaxy (assuming $\mathrm{H}_{\mathrm{o}}=75 \mathrm{~km} \mathrm{~s}^{-1} \mathrm{Mpc}^{-1}$ ). From these data Hintzen concluded that the A1775 system could not be a stable bound system, but either a superposition of two galaxies in two different clusters, or some sort of interaction. The latter conclusion seems to be supported by the discovery of O'Dea and Owen (1985) that the NW component is a wide-angle-tail source. Hayes (1982), using Palomar Schmidt plates modeled the cD system using the "standard" King (1966) models and found that both the stable bound model and the superposition ideas were both unlikely, leaving the collision model. We have now obtained broadband BVRI CCD images of the $\mathrm{CD}$, and it is this data that we present here.

\section{Observations}

The BVRI CCD images of the A1775 cD were obtained 10 June 1986 under the KPNO Request Observing Program. The \#1-0.9 m telescope was used in conjunction with the TI2 CCD. The f-ratio used was $\mathrm{f} / 7.5$, giving an image scale of $0.43^{\prime \prime}$ pixel ${ }^{-1}$. The seeing was mostly near 2 ". Initial flattening, etc., was done on the mountain while the actual reductions were done at STScI using the UNIX-based version of the "Vista" reduction package. The filters used are the KPNO standard broadband set. 


\section{Discussion}

The most remarkable feature of the $\mathrm{A} 1775 \mathrm{cD}$ is the unusual shape of the common envelope which surrounds the two nuclei. In all of our images it shows up as having a very square or boxy appearance - indeed, truncated. In Figure 1 we plot the $\mathrm{R}$ isophotes.

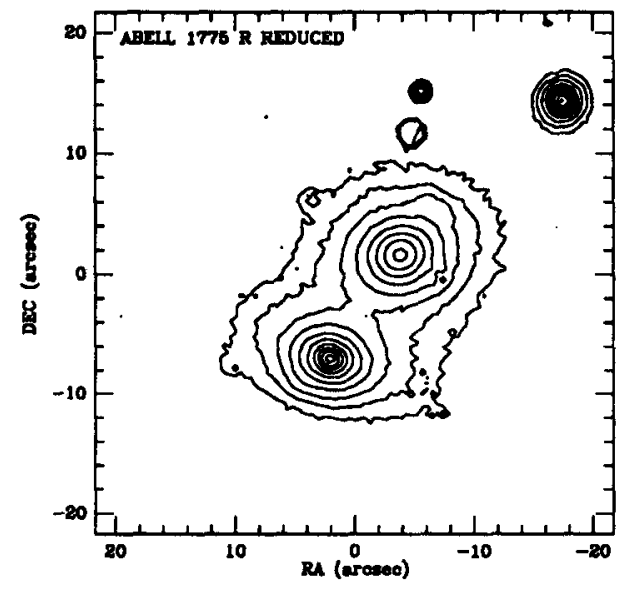

Figure 1: Half-magnitude $\mathbf{R}$ isophotes of the A1775 cD. North is at the top, East to the left

Indeed, there is a very steep fall off of light in the outer regions of the SE component which shows up in the brightness profiles of the system. Figure 2 is the $\mathrm{V}$ brightness profile at position angle $=125.3^{\circ}$. The profile shows that there is $30 \%$ more light between the two than one would expect for a simple superposition of two ellipticals, thus confirming one of Hayes' (1982) conclusions that the system is not a superpostion of two ellipticals.

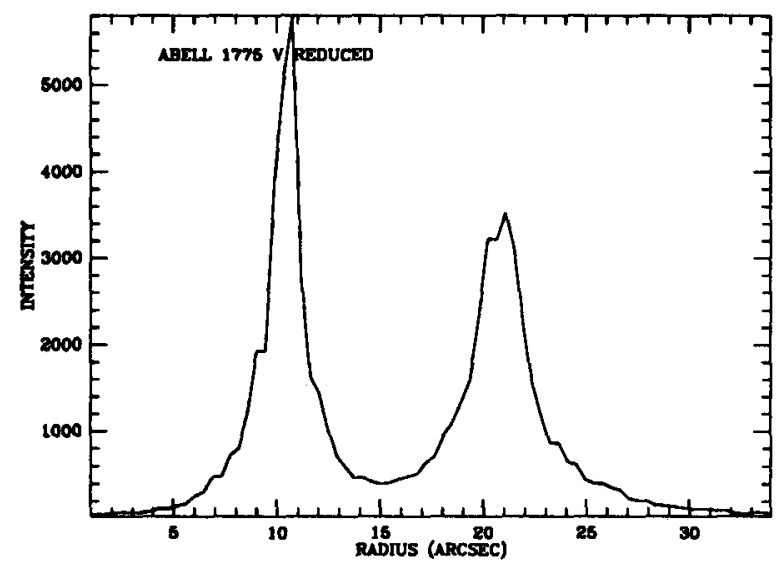

Figure 2: $\mathrm{V}$ band brightness profile of A1775. SE is to the left. Position angle of the cut is $125.3^{\circ}$.

In addition, the fainter (NW) component shows very strong isophotal twists and radial variations in ellipticity. This would seem to indicate that this component at least is suffering from some sort of perturbation. The brighter (SE) component also shows isophotal twists and ellipticity changes, but not to the same extent. We plot the isophotal twists and the variations in ellipticity in Figures 3 and 4 below. 

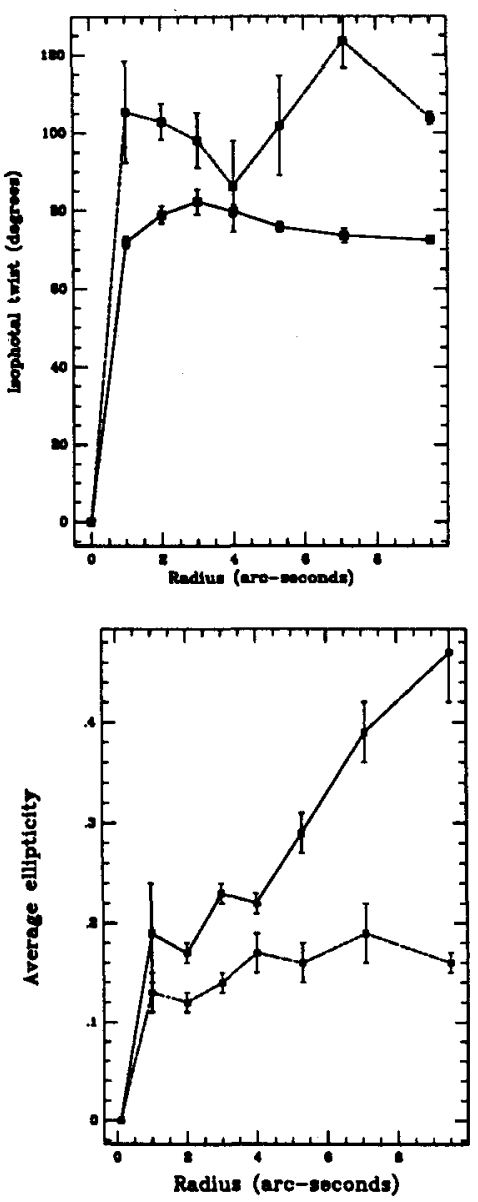

Figure 3: Average isophotal twists. The open symbols and solid line are for the SE (brighter) component, while the solid symbols and dotted line refer to the NW component.

Figure 4: Average ellipticity of the cD system. The symbols have the same meaning as in Figure 3.

We have also looked at the (B-V) colours of the two components. We find that for both components, (B-V) decreases (as expected for ellipticals) from 1.06 to about 0.80 . However, the gradient of the index is sharp; the colour drops $\approx 0.2$ magnitudes in only a few arc-seconds, supporting our notion that the profiles are being truncated by a collision. We see no evidence for star formation.

Other data come from looking at the brightness profiles and comparing them to the deVaucouleurs (1948) $r^{1 / 4}$ law. In Figure 5 we plot the surface brightness profiles for the two components in each of BVRI (in relative magnitudes) and a scaled deVaucouleurs law in each of the frames. One notices immediately that none of the profiles are well-fit by the deVaucouleurs model. In the outskirts of most of the profiles, the model is too faint by a factor of 0.2 magnitudes.

Our last data comes from looking at the residuals of models constructed using our photometric fits subtracted from theobserved data. We see that the SE component is fairly well modeled (except for the core, which is expected as we have not deconvolved the seeing from the images), but there is a great deal of low luminosity diffuse brightness left over from the NW component, indicating that this component is not being well-modeled after all. 

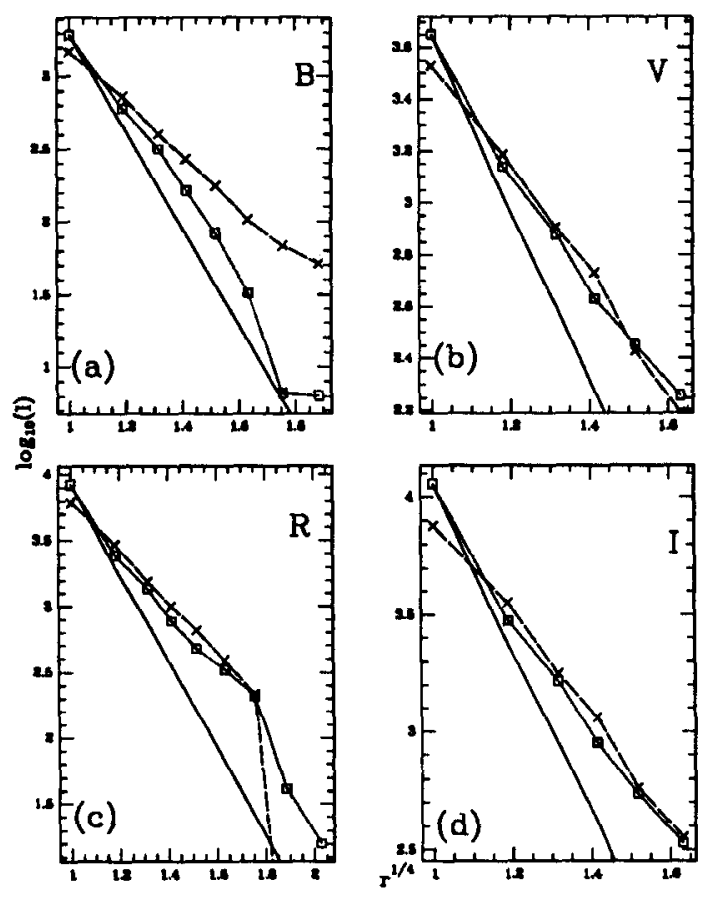

Figure 5: Brightness profiles of A1775. The dotted line is the SE component; the dashed line the NW component, and the solid line is the $r^{1 / 4}$ law. We see that in virtually all cases, the observed profiles are brighter than the model. Subfigure (a) is the $\mathrm{B}$ data; (b) the V data; (c) is the R data, and (d) is the I data. Overall the data fits the model quite poorly, something expected for a collision.

We believe that this is caused by the effects of a collision, which is being seen in the NW component more because it is about half the mass of the $\mathrm{SE}$ component (see Conclusions below). In Figure 6 we plot the residual contours for the I filter data.

Figure 6: Residual contours of I filter data. Note the large, diffuse area near the posi-

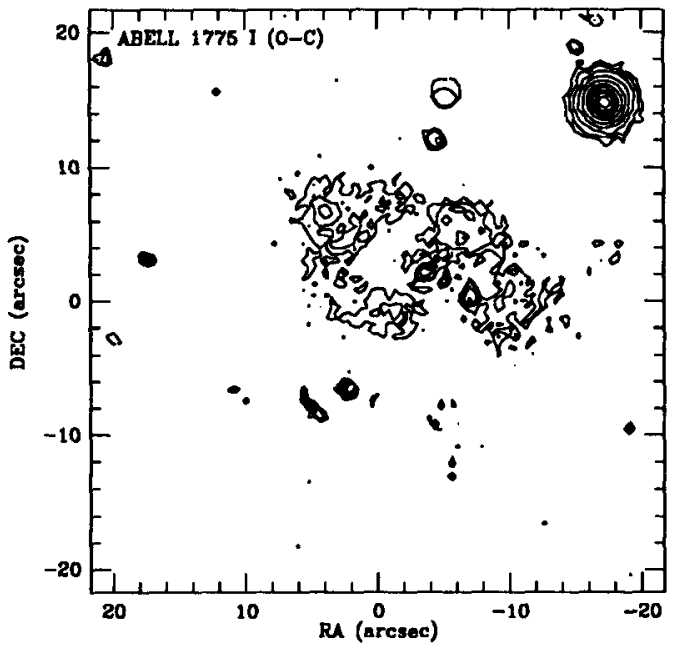
tion of the NW component, showing that the model is not adequate, and we believe is indicative of a collision. Both cores are not well-modeled as we have not deconvolved the seeing from the data.

\section{Conclusions}

From the isophotal structure, previous measures of the relative velocities of the two components (Chincarini et al. (1971), Jenner (1974) and Huchra (1982)), and the radio morphology mentioned above, we believe that the bound stable model proposed by Chincarini et al. is not 
tenable. Also, looking at the the (B-V) colour index we see that the colour gradient is steep, but that the range in (B-V) (from about 1.06 to about 0.8 ) is typical for normal elliptical galaxies. The brightness profile of the two galaxies is also diagnostic, in that the amount of light coming from between them is more than one would expect if the $\mathrm{cD}$ was a simple superposition of two galaxies that followed the "standard" King model in brightness (see also Hayes (1982)). On the basis of this data, we believe that the idea that the $\mathrm{cD}$ is a simple superposition can also be ruled out.

This leaves us with a collision as the best model left for A1775. The steepness of the colour gradients for both components, the boxy-shaped halo, the very poor fits by the deVaucouleurs $r^{1 / 4}$ law to the brightness profiles, and the residual brightness from the model fitting are all evidence for an unusual interaction. It appears that both components are strongly affected by each other, and that there has been some severe modification of the normal brightness profile (assuming the $r^{1 / 4}$ law is universal in all enviroments). Also of interest are the isophotal twists because while the outer isophotes of the NW component do point towards the SE component, the SE component's isophotes do not seem to point to the NW (see Figure 1). In this case we believe we may be seeing the effects of the difference in mass between the two components. The SE component's core is about twice as bright as the NW component's core in all colours. If we assume a constant $\mathrm{M} / \mathrm{L}$ ratio for both galaxies, then the SE component is about twice the mass of the NW component. Therefore, the NW component of the pair would manifest the effects of the collision first and to a greater degree that the more massive SE partner. One is inclined to point out that the high relative velocities between the two components (about 1700 $\mathrm{km} \mathrm{s}^{-1}$ ) is an argument against the galaxies being in the same cluster. However, both A98 and A2152 have cluster velocity dispersions which are roughly the same as difference in velocities of the two components in A1775 (Noonan 1981), so it seems reasonable to assume that the two components are in the same cluster. Therefore, we are probably seeing a high speed collision of two unequal mass ellipticals.

Finally, we would like to thank George Jacoby at NOAO who took the CCD images.

\section{REFERENCES}

Chincarini, G., Rood, H.J., Sastry, G.N., Welch, G.A.; (1971) Ap. J., 168, 11 deVaucouleurs, G.; (1948) Ann. d'Ap., 11, 247

Hayes, J.J.E.; (1982) unpublished M.Sc. thesis, Saint Mary's University, Halifax

Hintzen, P.; (1979) Pub. A.S.P., 91, 426

Huchra, J.P.; (1982) - private communication

Jenner, D.C.; (1974) Ap. J., 191, 55

King, I.R.; (1966) Astron. J., 71, 64

Miley, G. and Harris D.; (1977) Astr. Ap (Letters)., 61, L23

Noonan, T.W.; (1981) Ap. J. Supp. Ser., 45, 613

O'Dea, C.P. and Owen, F.N.; (1985) Astron. J., 90, 927 